\title{
Optical Sinc-Shaped Nyquist Pulse Source Based on a Single Mach-Zehnder Modulator
}

\author{
Jianqi Hu, Simon J. Fabbri, and Camille-Sophie Brès \\ Ecole Polytechnique Fédérale de Lausanne (EPFL), Photonic Systems Laboratory (PHOSL), \\ Station 11, STI-IEL, CH-1015 Lausanne, Switzerland \\ Tel: +41(21)69375 90, e-mail: simon.fabbri@epfl.ch
}

\begin{abstract}
We present a precise and flexible Sinc-shaped Nyquist pulse source based on a single Mach-Zehnder modulator. The use of multi-harmonic RF signals provides direct control on the Nyquist pulse train generation. The variable comb source is demonstrated by selecting the number of RF components used to drive the optical modulator, leading from 3 to $9 \mathrm{comb}$ lines. Furthermore, different operating points are reported to show the multi-harmonic comb generator potential to provide near-perfect Nyquist pulse source with pure rectangular optical spectrum or with minimized insertion loss.
\end{abstract}

Keywords: Sinc-shaped Nyquist pulse, optical frequency comb, electro-optic modulation.

\section{INTRODUCTION}

Generation of Sinc-shaped optical Nyquist pulses is essential for a wide range of applications in optical telecommunications, RF photonics, and optical signal processing. Indeed, due to its rectangular spectrum and zero inter-symbol interference (ISI) in time domain, the newly developed optical orthogonal time division multiplexing (O-OTDM) and Nyquist WDM technique [1] can achieve comparable spectral efficiency as optical orthogonal frequency division multiplexing (O-OFDM), while the receiver complexity is reduced and it is less sensitive to the nonlinearities of the link. Besides, since the Nyquist pulse train corresponds to a number of optical frequency combs in spectrum with equal amplitude and linear spectral phase, it can be utilized to implement optical or microwave filters with rectangular spectrum [2]. In addition, all-optical sampling using Nyquist pulses has recently been demonstrated based on external modulators with tunable sampling speed [3], thereby avoiding the need for nonlinear mixing with short optical pulse as the gating function.

Therefore, considerable efforts have been placed to generate Sinc-shaped Nyquist pulses with a variety of methods [4-8]. By cascading two Mach-Zehnder modulators (MZM) with synchronized RF frequencies (f, 3f) to each of the modulator, near-ideal Sinc-shaped Nyquist pulses were obtained with the corresponding 9-line comb spectrum [4]. Sinc-shaped Nyquist pulses were also demonstrated using a single dual-parallel Mach-Zehnder modulator (DP-MZM) with 5 spectral comb lines [5]. In [6], by putting a segment of dispersion compensating fiber (DCF) in between a phase modulator (PM) and a MZM, serving as a modified lens, Nyquist pulses were generated with a 11-line comb spectrum after a selective bandpass filter. In order to expand the spectrum, and therefore narrowing the Nyquist pulse width, four-wave mixing technique were utilized with the help of a waveshaper to tailor the spectrum [7]. Indeed, liquid crystal spatial light modulator can be used to implement line-by-line shaping of mode locked laser spectrum or super-continuum comb source into Nyquist pulses, with the full control of the amplitudes and the relative phases [8]. In order to obtain flatter and wider optical combs, more than one RF clock were used in [9] to smooth the central region of the output spectrum from a single asymmetrical drive Mach-Zehnder. Although Nyquist pulses were not investigated in [10], rectangular spectrum optical frequency combs were demonstrated with a multiple harmonics electrical signal.

In this paper, we propose a novel method to generate Sinc-shaped Nyquist pulses using a single modulator driven by multi-harmonic RF signals. Near ideal Sinc-shaped Nyquist pulses with optical comb comprising of 3, 5,7 , and 9 lines are generated at the repetition rate of $10 \mathrm{GHz}$, by using from one to four clock sources, respectively. A single MZM is used so that the scheme is simple and the complexity is maintained in the electrical domain. This method is highly reconfigurable in terms of the number of comb lines $(3,5,7$, and 9$)$ as well as the repetition rate. Furthermore, due to the multi-harmonic nature of the driving RF signal, the electrical power required is lower than the standard techniques to generate optical combs using external modulators. In addition, we also demonstrate the trade-off between the side-mode suppression ratio (SMSR) of the optical comb and the insertion loss due to the modulation in this method.

\section{MULTI-HARMONIC BASED SINC-SHAPED PULSE SOURCE}

The conceptual setup for multi-harmonic optical Sinc-shaped Nyquist pulses is shown in Fig. 1. Suppose $N$ RF clock sources with incremental frequencies, i.e. $\omega_{m}, 2 \omega_{m}, \cdots, N \omega_{m}$, are used to modulate the MZM with the same modulation index, the output electrical field from MZM can be written as, 


$$
E_{\text {out }}(t)=\cos \left(\varphi+\beta \sum_{k=1}^{N} \sin \left(k \omega_{m} t\right)\right) e^{i \omega_{0} t}=\left\{\cos \varphi \cos \left(\beta \sum_{k=1}^{N} \sin \left(k \omega_{m} t\right)\right)-\sin \varphi \sin \left(\beta \sum_{k=1}^{N} \sin \left(k \omega_{m} t\right)\right)\right\} e^{i \omega_{0} t}
$$

where $\varphi=\pi V_{\text {bias }} / V_{\pi_{0}}$ and $\beta=\pi V_{k} / 2 V_{\pi_{k}}(k=1,2, \cdots, N)$ is the modulation index and is kept constant for all the RF frequencies. $V_{\pi_{0}}$ and $V_{\pi_{k}}(k=1,2, \cdots, N)$ are the half-wave voltage of the MZM for DC and RF angular frequencies $\omega_{m}, 2 \omega_{m}, \cdots, N \omega_{m}$, respectively. If the modulation index $\beta$ is small, Eq. (1) can be approximated by,

$$
E_{\text {out }}(t) \approx\left\{\cos \varphi-\frac{\beta \sin \varphi}{2} \sum_{k=1}^{N}\left(e^{i k \omega_{m} t}+e^{-i k \omega_{m} t}\right)\right\} e^{i \omega_{0} t}
$$

Therefore, $2 N+1$ comb lines centered at $\omega_{0}$ and spaced by $\omega_{m}$ are generated at the output of the MZM. In order to generate Sinc-shaped Nyquist pulses, equal amplitude and equal phase of the different frequency components must be satisfied, i.e.

$$
\cos \varphi=-\frac{\beta \sin \varphi}{2}
$$

Since the modulation index $\beta$ is relatively small, $\varphi$ is close to $-\pi / 2$. The bias point of MZM is close to the minimum transmission point (MITP), and this is in accordance with the small signal modulation condition. Hence, while the complete harmonic analysis can be carried out for Equation (1) with Taylor expansion, it is not necessary in this scenario. Sinc-shaped pulse meeting the Nyquist criteria can therefore be generated using small amplitude RF signals along with a phase control of the RF components.

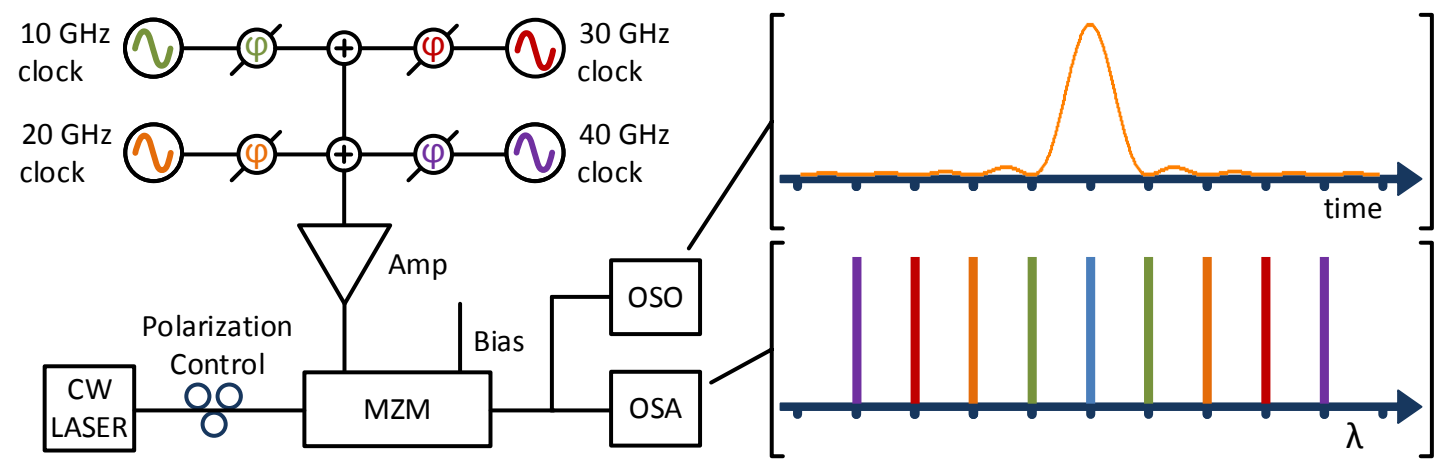

Figure 1. Multi-harmonic comb based Nyquist pulse source concept. Left: schematic of a MZM driven by a set of RF sources. Right: corresponding spectrum and waveform when Nyquist conditions are obtained. OSO: Optical Sampling Oscilloscope, OSA: Optical Spectrum Analyzer, $\varphi$ : tunable phase control.

\section{EXPERIMENTAL RESULTS}

The implementation of the Nyquist pulse source shown in Fig. 1 was based on a commercial low $V_{\pi}$ MZM. The modulator used for the experiment has a $30 \mathrm{GHz}$ electrical bandwidth, an extinction ratio of $30 \mathrm{~dB}$, and an insertion loss of $4.2 \mathrm{~dB}$. A narrow linewidth laser tuned at $1549.79 \mathrm{~nm}$ was providing $13 \mathrm{dBm}$ of optical power to the MZM. The output of the comb source was directed towards the time and spectrum measurement equipment using a 50:50 coupler. The time trace was acquired using a $500 \mathrm{GHz}$ bandwidth optical oscilloscope (OSO). The optical power input to the OSO was maintained constant at $3.8 \mathrm{dBm}$ for all cases with the help of an EDFA. The based clock was set at $10 \mathrm{GHz}$, and hence the higher harmonics used were 20, 30, and $40 \mathrm{GHz}$. In the implementation reported here, only three RF clock sources had been used in the experiment. Indeed the third order harmonic was generated from a narrow-band frequency tripler having a $10 \mathrm{GHz}$ signal as input and a $30 \mathrm{GHz}$ signal at its output. The phase of each electrical components was controlled independently either from the clock source directly or with the insertion of tunable electrical delay line in the path.

Figure 2 presents the Sinc-shaped Nyquist pulses obtained experimentally for different number of harmonics forming the driving RF signals, from a single frequency signal to four RF components with equal spacing. The direct control of both the RF power and the relative phase of these components allowed for the generation of Nyquist pulses. Indeed, each pair of sidebands was directly synthesized by the corresponding RF component. In addition, the DC bias voltage predominantly controls the power of the central carrier. The resulting optical combs obtained was characterized with minimal flatness, measured at 0.02, 0.07, 0.09, and $0.12 \mathrm{~dB}$ for the 3-, 5-, 7-, and 9-line comb, respectively. Figure 2 highlights the rectangular shape of the optical combs of all cases. Despite a limiting optical spectrum analyser resolution $(0.02 \mathrm{~nm})$, the side-mode suppression ratio can be measured at least greater than $28.5 \mathrm{~dB}$, giving potential for Nyquist channel multiplexing with minimum interference on adjacent channels [11]. The Sinc-shaped pulse obtained show the clear main peak followed by the subsequent symmetric side lobes indicating the alignment of the relative phase. Due to the nature of the 
method presented here, long time stability had been observed while the main source of drift would be the temperature variation induced modulator's bias shift. In the case of the four harmonics forming the RF signals, the pulse obtained had a width of 22.2 ps zero crossing and a repetition rate of $10 \mathrm{GHz}$. And all the experimental pulses were in good match with ideal Sinc-shaped Nyquist pulses.
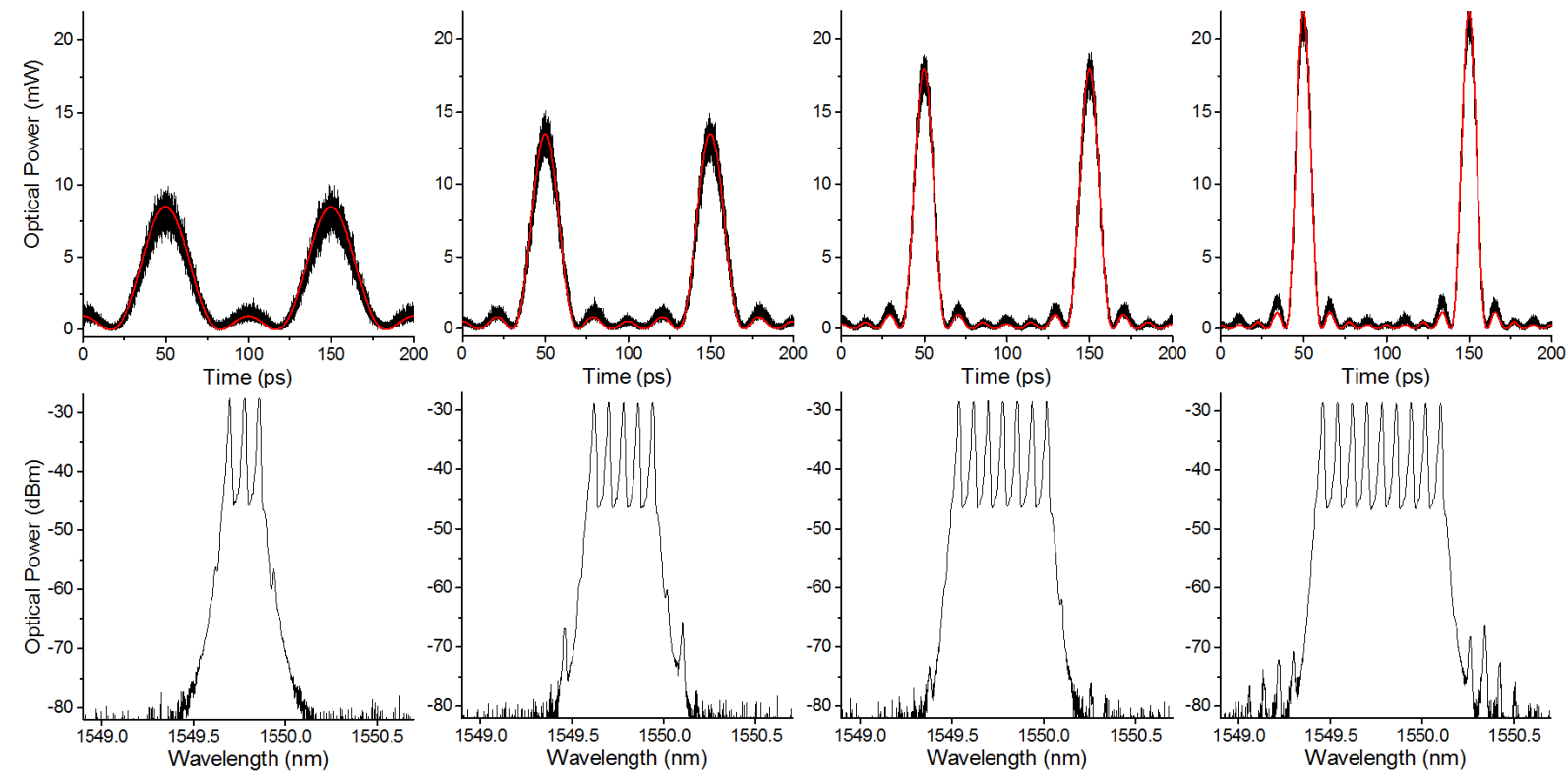

Figure 2. Optical pulses and optical comb spectra for 1, 2, 3, and 4 RF frequencies driving signal, respectively. Red: Simulated ideal Sinc-shaped Nyquist pulses.

Figure 3 demonstrated the variations in the waveform as well as the spectrum for different RF signal power level in the case of the 9-line comb. Higher driving voltage being applied to a MZM increases the optical power of the sidebands. But it inevitably increase the secondary sidebands and hence reduces the SMSR of the comb. Interesting trade-off was studied as a lower RF signal provides a greater SMSR but more insertion loss whereas a higher RF signal results in higher average power at the output of the pulse source but degrade the SMSR. At high RF power level, which is not shown in this paper, the pulse is heavily distorted due to the contribution of secondary and tertiary sideband pairs. This would lead to the frequency interference from different RF signals inside the rectangular spectrum and also the higher order harmonics outside of the rectangular spectrum.
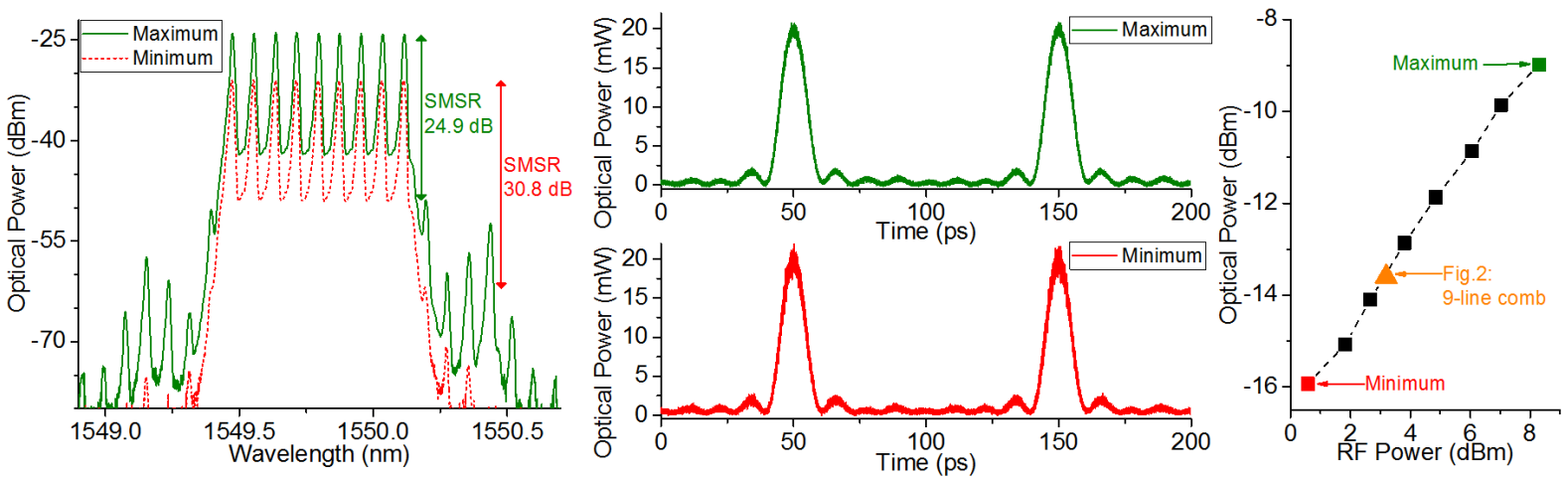

Figure 3. Left: Optical spectra of the 9-line comb with the minimum (red) and maximum (black) RF driving power. Center: Nyquist pulses for the two cases. Right: Average optical power at the output of the comb source versus total electrical power of the RF driving signal.

Over the range of RF power available for the experiment reported here, the Nyquist pulse shape was maintained for all the cases. However, for lower RF driving signal power, although the SMSR is higher, the optical signal input to the EDFA for being detected by OSO is lower as well. Therefore, excessive noise is amplified in this condition, despite $5 \mathrm{~nm}$ optical filter was used to filter out the amplified spontaneous emission (ASE). Indeed, the results presented in Fig. 3 are well illustrating the trade-off of the multi-harmonic comb source as a lower RF driving signal results in excessive ASE due to large insertion loss. On the other hand, a higher RF driving signals result in a greater power per comb line but decrease the SMSR, thereby would give penalty in the case of super-channel multiplexing. 


\section{CONCLUSIONS}

In this paper, we experimentally demonstrated a simple and compact system to generate near-perfect optical Sinc-shaped pulse, matching the Nyquist requirement. Emphasis is given to the inherent flexibility of the comb source proposed by showing the range of pulse width while maintaining the repetition rate constant. Furthermore, the transition from low RF power offering clean comb spectrum to medium RF power lowering the insertion loss of the comb source is presented, with near-perfect Nyquist pulses in all cases.

\section{ACKNOWLEDGEMENTS}

This work was supported by the Swiss National Science Foundation under Grant 159897.

\section{REFERENCES}

[1] D. Hillerkuss et al.: Single-laser 32.5 Tbit/s Nyquist WDM transmission, J. Opt. Commun. Netw., vol. 4, no. 10 , pp. $715-723,2012$.

[2] V. R. Supradeepa et al.: Comb-based radiofrequency photonic filters with rapid tunability and high selectivity, Nat. Photonics, vol. 6, no. 3, pp. 186-194, 2012.

[3] S. Preussler et al.: Frequency-time coherence for all-optical sampling without optical pulse source, Sci. Rep., vol. 6, 34500, 2016.

[4] M. A. Soto et al.: Optical Sinc-shaped Nyquist pulses of exceptional quality, Nat. Commun., vol. 4, 2898, 2013.

[5] Q. Wang et al:: Ultra-flat optical frequency comb generator using a single-driven dual-parallel MachZehnder modulator, Opt. Lett., vol. 39, no. 10, pp. 3050-3053, 2014.

[6] D. Wang et al.: Optical Nyquist pulse generation using a time lens with spectral slicing, Opt. Exp., vol. 23, no. 4, pp. 4329-4339, 2015.

[7] S. Cordette et al:: Bandwidth and repetition rate programmable Nyquist Sinc-shaped pulse train source based on intensity modulators and four-wave mixing, Opt. Lett., vol. 39, no. 23, pp. 6668-6671, 2014.

[8] H. Nguyen Tan et al.: No-guard-band wavelength translation of Nyquist OTDM-WDM signal for spectral defragmentation in an elastic add-drop node, Opt. Lett., vol. 38, no. 17, pp. 3287-3290, 2013.

[9] N. Yokota et al:: Harmonic superposition for tailored optical frequency comb generation by Mach-Zehnder modulator, Opt. Lett., vol. 41, no. 5, pp. 1026-1029, 2016.

[10] S. Fabbri et al.: Multi-harmonic optical comb generation, in Proc. ECOC, 2012, paper Mo.2.A.2.

[11] M. A. Shoaie et al:: A simple all-optical format transparent time and wavelength demultiplexing technique for WDM \& orthogonal-TDM Nyquist channels, in Proc. ECOC, 2015. 